Cahiers Charlevoix

\title{
Roger Bernard, intellectuel de l'Ontario français
}

\section{Yves Frenette}

Volume 5, 2002

URI : https://id.erudit.org/iderudit/1039350ar

DOI : https://doi.org/10.7202/1039350ar

Aller au sommaire du numéro

\section{Éditeur(s)}

Société Charlevoix

Presses de l'Université d’Ottawa

ISSN

1203-4371 (imprimé)

2371-6878 (numérique)

Découvrir la revue

Citer ce document

Frenette, Y. (2002). Roger Bernard, intellectuel de l'Ontario français. Cahiers Charlevoix, 5, 11-43. https://doi.org/10.7202/1039350ar

\section{Résumé de l'article}

Yves Frenette, professeur au département d'Histoire, du Collège universitaire Glendon de Toronto, brosse la carrière de Roger Bernard dans un article-hommage à notre premier membre disparu. Dans son panorama, il rappelle quel intellectuel engagé il était et combien dérangeait la thèse, qu'il avait conçue, de l'émergence d'une identité bilingue dans la francophonie minoritaire. À travers les écrits du sociologue, notamment De Québécois à Ontarois, l'enquête Vision d'avenir, et ses articles sur l'exogamie et les transferts linguistiques publiés dans nos cahiers, comme ses travaux pionniers sur les sentiers migratoires, tels qu'ils se manifestent dans Le Travail et l'espoir, Frenette retrace la genèse et le cheminement de cette pensée, sans condescendance cependant, avec ses forces et ses faiblesses, en signalant surtout la réflexion et les discussions que cet « intellectuel de l'Ontario français » aura su provoquer. Une bibliographie de l'oeuvre de Roger Bernard accompagne cet article. 


\section{Hommage}




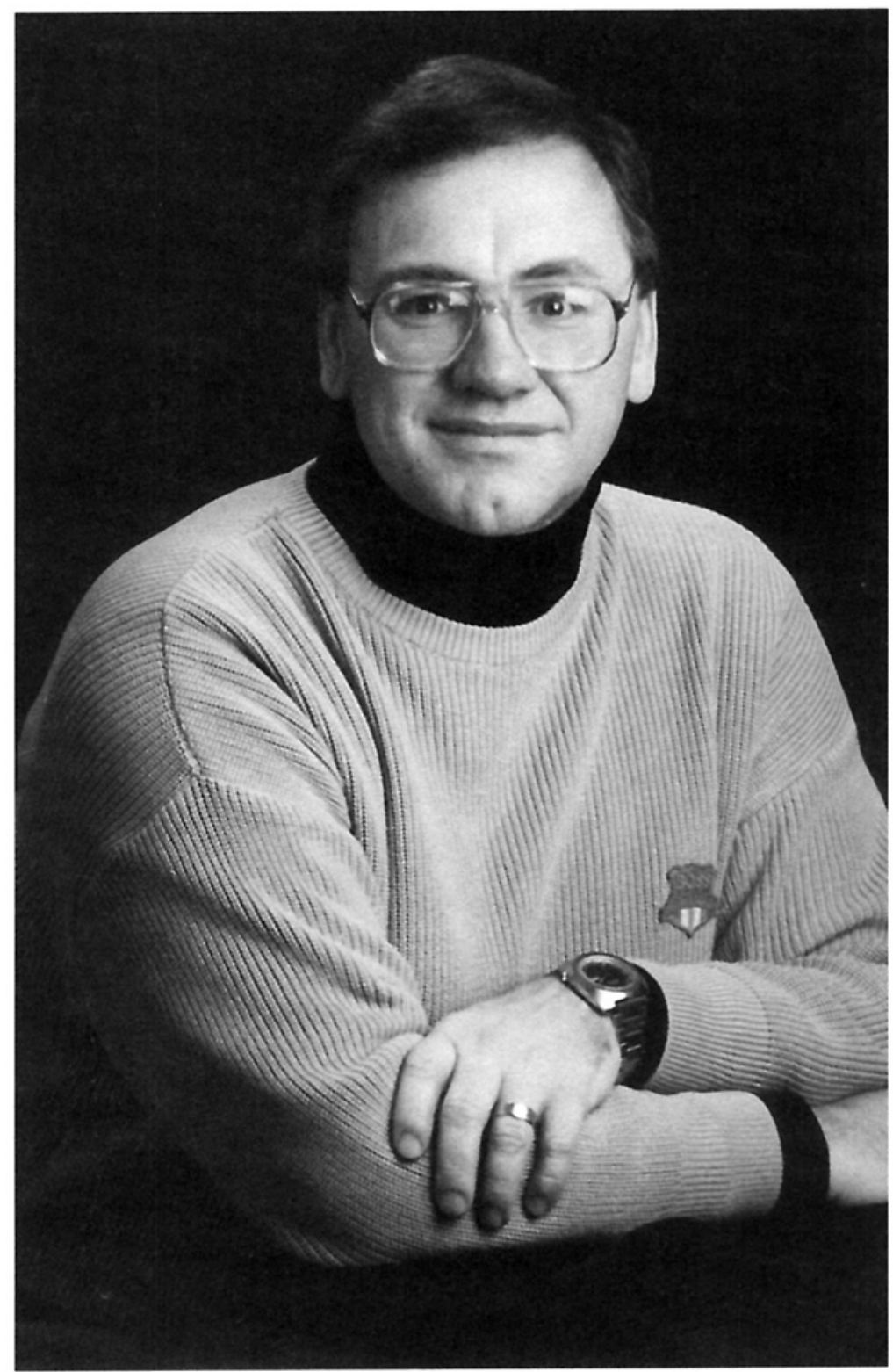

ROGER BERNARD 


\title{
ROGER BERNARD, INTELLECTUEL DE L'ONTARIO FRANÇAIS
}

\author{
Yves Frenette
}

Département d'histoire Collège universitaire Glendon 


\section{SOMMAIRE}

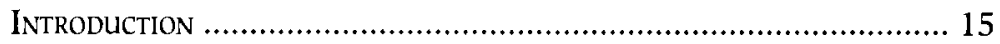

I - LA GENĖSE D'UNE PENSÉE ............................................... 17

II - De Québécois À OntaroIs ..................................................... 19

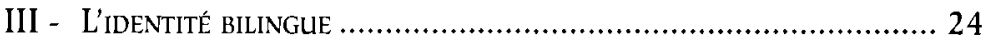

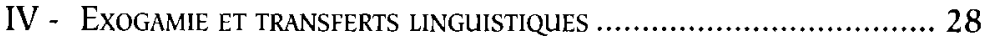

V - LE SPÉCIALISTE DES MIGRATIONS ………….................................. 30

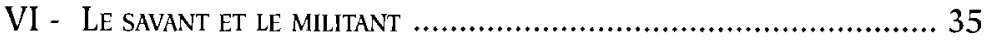

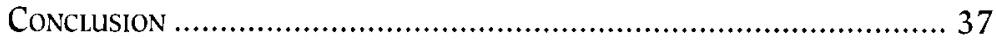

L'CEuvre de Roger Bernard ....................................................... 39 


\section{ROGER BERNARD,}

INTELLECTUEL DE L'ONTARIO FRANÇAIS

\section{INTRODUCTION}

«Je poursuivrai inlassablement mon travail intellectuel jusqu'à la fin de ma carrière en retenant l'hypothèse que je contribue peut-être à l'invention intellectuelle ${ }^{1}$." C'est par ces mots que le sociologue Roger Bernard concluait en 1995 une réflexion sur la pratique de la recherche en Ontario français. Cinq ans plus tard, il décédait subitement, la Grande Faucheuse venant le surprendre au moment où il s'apprêtait à contribuer à l'invention intellectuelle d'une autre façon qu'il l'avait fait jusque-là. En effet, il voulait se lancer dans une aventure d'écriture de nature intimiste en rédigeant un livre fondé sur des carnets de notes noircis lors d'un voyage en France au printemps de $1998^{2}$. Déjà, en 1994, il nous avait confié être un peu fatigué de creuser les mêmes ornières.

Mais le destin en décida autrement et l'Ontario français se souviendra de Roger Bernard comme d'un intellectuel

\footnotetext{
${ }^{1}$ Roger Bernard, "Réflexions d'un chercheur», dans Jacques Cotnam et al., dir., La Francophonie ontarienne. Bilan et perspectives de recherche, Ottawa, Le Nordir, 1995, p. 339.

${ }^{2}$ Robert Yergeau, "Avant-propos", Roger Bernard, À la défense de Montfort, Ottawa, Le Nordir, 2000, p. 7.
} 
engagé dans l'élaboration d'une thèse capitale pour les études franco-ontariennes et canadiennes-françaises, celle de l'émergence d'une identité bilingue au sein de la société franco-ontarienne et des autres minorités de langue française dispersées sur le territoire canadien. Qualifiée de pessimiste par les uns et de réaliste par les autres, la thèse de l'identité bilingue et son corollaire, la "secondarisation" de la langue française, ont fait de Roger Bernard un Cassandre dans certains milieux. On l'a accusé d'être un "déterministe» qui ne regardait que les chiffres. C'est là déformer une pensée riche, complexe, paradoxale même.

Roger Bernard dérangeait, en particulier les militants qui pensaient avec les "tripes" et qui accusaient leurs compatriotes d'être indifférents envers la langue et la culture françaises. Pour lui, les Franco-Ontariens exhibaient un comportement rationnel, explicable par l'analyse théorique. Celle-ci était seule garante d'une action lucide: "À l'étape de la refrancisation, les gens d'action doivent prendre le temps de poser les bonnes questions s'ils espèrent trouver des réponses satisfaisantes et proposer des stratégies valables. L'action efficace passe par une réflexion sérieuse ${ }^{3} . »$ En fait, Bernard aimait déranger et il y avait chez lui un côté pamphlétaire, comme en font foi ses critiques à l'égard de ses collègues chercheurs, qu'il jugeait pusillanimes:

Procéder à un bilan critique [. .] est probablement une entreprise périlleuse qui risque de décupler le nombre de mes détracteurs: ceux-ci n'hésiteront pas à relancer l'attaque. Ce sera de bonne guerre! Du choc des idées, des idéologies et des clans, surgiront sûrement des problématiques nouvelles, qui alimenteront les recherches des jeunes chercheurs. Assez de complaisance et de courbettes! Redressons-nous! L'heure est aux défis, des défis méthodologiques et intellectuels. Fini le misérabilisme de ces pauvres chercheurs des communautés minoritaires, qui sont supposément pris entre l'arbre et l'écorce $[. .]^{4}$.

\footnotetext{
${ }^{3}$ Roger Bernard, De Québécois à Ontarois. La communauté franco-ontarienne, Hearst, Le Nordir, 1988, pp. 10-11.

${ }^{4}$ Id., "Réflexions d'un chercheur», p. 327.
} 
Roger BERNARd, INTELLECTUEL DE L'ONTARIO FRANÇAIS

\section{I - LA GENÈSE d'UNE PENSÉE}

Originaire de Departure-Lake, près de Cochrane, où il naît en 1944, Roger Bernard fait ses études secondaires au Petit Séminaire de Hearst. Sous l'influence d'un professeur, il découvre alors Jean-Paul Sartre, Karl Marx, et il prend conscience que «le monde était une construction sociale $^{5} »$. Il lit beaucoup, surtout des essais de combat, tel le Nègres blancs d'Amérique de Pierre Vallières. Inscrit au baccalauréat en lettres françaises, il fréquente aussi les grands écrivains, y compris Marcel Proust, qu'il adore.

Muni de son diplôme, Bernard enseigne au palier secondaire pendant l'année 1968-1969, avant de revenir aux études, cette fois au Collège Glendon de l'université York. Encouragé par le professeur Jean Burnet, il obtient une maîtrise en sociologie en 1971. Ces années à Glendon sont cruciales: Bernard continue à s'intéresser à la question de la décolonisation, il prend connaissance du rapport de la Commission Laurendeau-Dunton et il est bouleversé par la Crise d'octobre de 1970. Pour la première fois, il porte intérêt aux Franco-Ontariens, qu'il voit comme un peuple colonisé et exploité.

En 1972, Bernard retourne dans le Nord et devient professeur au Collège universitaire de Hearst, où il occupe aussi plusieurs postes administratifs. Il a beaucoup de plaisir à enseigner, mais, de tempérament inquiet, il vit chaque début de session avec fébrilité. Il considère comme «une tâche presque sacrée de former des jeunes gens, de les mener plus loin dans leur cheminement intellectuel et humain ${ }^{6} \%$. Il observe le milieu où il vit. Ayant en tête les recherches réalisées pour la Commission Laurendeau-Dunton, ainsi que les écrits des sociologues Raymond Breton et Danielle Juteau-Lee, il est fasciné par

${ }^{5} L$ 'Ontario se raconte. De A à $X$. Entrevues radiophoniques réalisées et présentées par François-Xavier Chamberland, Toronto, Éditions du Gref, 1999, p. 415.

${ }^{6}$ Yergeau, op. cit., p. 10. 
les comportements linguistiques de ses compatriotes du Nord et décide d'y consacrer un autre mémoire de maîtrise, qui sera soutenu à l'Université d'Ottawa en $1978^{7}$.

Les recherches qu'il effectue alors sont cruciales dans l'élaboration de sa pensée, puisqu'il développe des idées qui deviendront la base de ses écrits ultérieurs. En effectuant des entrevues à Hearst, à Kapuskasing et à Cochrane, Bernard constate que, à l'extérieur du foyer, de l'église paroissiale et de l'école élémentaire, les Franco-Ontariens montrent une nette propension à utiliser l'anglais, dans un contexte où les médias de langue anglaise dominent. Toutefois, en y regardant de plus près, il se rend compte que les individus nés au Québec emploient le français plus souvent que ceux nés en Ontario. Surtout, il peut établir des corrélations entre le contexte démographique et la langue d'usage: plus les francophones sont minoritaires, plus ils utilisent l'anglais dans leur vie quotidienne; l'anglicisation est ainsi plus poussée à Cochrane, qui compte $44,5 \%$ de francophones, qu'à Kapuskasing, qui en compte $57,7 \%$, et elle est beaucoup moins avancée à Hearst, où les francophones représentent $80 \%$ de la population. Dans les trois villes, cependant, les Franco-Ontariens interviewés par Bernard s'entendent sur le fait qu'être bilingue constitue l'élément essentiel de leur culture.

Enseignant, chercheur et administrateur, Roger Bernard ressent toutefois un malaise, étant incapable d'assumer son rôle d'intellectuel dans un milieu qui, à l'extérieur des murs du collège, «n'en avait cure ${ }^{8}$ ». Bernard sera "guéri» lors de ses études doctorales à l'université McGill au début des années 1980. En côtoyant des intellectuels comme

\footnotetext{
${ }^{7}$ Roger Bernard, Le Comportement linguistique des Canadiens français de trois villes du nord-est de l'Ontario, Mémoire de maitrise (sociologie), Université d'Ottawa, 1978, 179 p.

${ }^{8}$ Yergeau, op. cit., p. 8. Le malaise de Bernard n'est pas sans rappeler celui du sociologue québécois Fernand Dumont. Voir son Récit d'une émigration. Mémoires, Montréal, Boréal, 1997, 268 p.
} 
ROGER BERNARD, INTELLECTUEL DE L'ONTARIO FRANÇAIS

Walter Moser et Monique Verney, il reconnaît que le travail intellectuel est aussi légitime que le travail physique.

\section{II - De Québécois à Ontarois}

Les années "nordiques» de Roger Bernard produisent donc une idée maîtresse. Celle-ci s'exprime d'abord à travers un essai, De Québécois à Ontarois, qui devient rapidement un classique en études franco-ontariennes. Outre l'influence des Breton, Juteau-Lee et autres experts des phénomènes ethniques, on y voit nettement esquissés les principes développés par les deux grands maîtres de la sociologie, Émile Durkheim et Max Weber. Bernard emprunte aussi des éléments et des méthodes à plusieurs autres sociologues, notamment Pierre Bourdieu et Alain Touraine, et il s'appuie sur une masse de données statistiques qu'il rend en 45 pages de tableaux et d'annexes.

Dès l'introduction, il annonce ses couleurs: «L'impasse qui guette les projets d'épanouissement culturel et linguistique de la communauté franco-ontarienne témoigne d'une continuité à travers l'histoire qui nous invite à l'analyse des situations qui perpétuent ce cul-de-sac ${ }^{9}$." Dans le premier chapitre, il analyse les bases de la communauté francoontarienne en reprenant les éléments théoriques d'un article publié en $1985^{10}$. Il compare ensuite les Franco-Ontariens aux Québécois. La résurgence de ces derniers s'accompagne d'un projet sociopolitique exigeant une redéfinition des Franco-Ontariens. Utilisant la théorie des conflits de Ralf Dahrendorf, Bernard étudie les changements qui entraînent des conditions différentes pour les francophones des deux groupes. Ainsi, au Québec, la segmentation ethnique et structurelle est plus poussée qu'en Ontario; en conséquence, les Québécois ont à leur disposition un réseau institutionnel

\footnotetext{
${ }^{9}$ Bernard, De Québécois à Ontarois, p. 10.

${ }^{10}$ Id., "L'Ontario français. Pratiques ethniques et théories sociologiques", Revue de l'Université d'Ottawa, vol. 55, n² 2 (avril-juin 1985), pp. 137-150.
} 
complet qui s'étend jusqu'au gouvernement et à la fonction publique, alors que les Franco-Ontariens sont forcés de partager des institutions avec la majorité de langue anglaise, «de telle sorte que leurs interactions se font aussi bien à l'intérieur du groupe ethnique qu'à l'extérieur ${ }^{11}$ ". De plus, les inégalités socio-économiques avec les anglophones ne sont pas aussi marquées en Ontario. Contrairement aux Québécois, les Franco-Ontariens mettent leur espoir dans un Canada bilingue et biculturel. De toute façon, étant minoritaires, ils ne sont pas en position de politiser les conflits ethniques. En outre, l'interaction quotidienne avec les anglophones favorise l'acculturation et les transferts linguistiques.

Clin d'œil à l'ouvrage classique d'Everett Hughes, le troisième chapitre s'intitule «Rencontre de deux mondes". Bernard explique comment le peuplement de l'Ontario par des Québécois influence la situation contemporaine des Franco-Ontariens. En niant le passé, commente-t-il, «nous refusons l'explication des trajectoires sociales francoontariennes déterminées par l'histoire que nous produisons et l'habitus que nous reproduisons depuis le début de notre histoire ${ }^{12} »$. Pour étudier la migration canadiennefrançaise, il faut dépasser les cadres étroits de la conquête du sol et de l'idéologie agriculturiste, et observer l'ensemble des migrations vers les trois grandes régions de la province. Il faut aussi comprendre les migrations interrégionales ayant cours à l'intérieur de l'Ontario, car elles amènent les francophones à vivre de plus en plus à la croisée de deux mondes: l'un anglais, l'autre français.

Dans la société rurale et traditionnelle, l'Église et la famille assuraient le maintien des idéologies de survie et d'épanouissement de la langue et de la culture françaises. Le francophone de l'Ontario vivait habituellement dans un

"Id, De Québécois à Ontarois, p. 31.

${ }^{12}$ Ibid., p. 40. 
village relativement homogène et majoritairement composé de Canadiens français. Les relations avec les individus d'autres groupes étaient limitées et les possibilités d'assimilation linguistique étaient minces. Le réseau institutionnel de langue française était presque complet. «Dans ce contexte, la culture française n'est pas menacée, et la volonté de vivre en français en Ontario s'inscrit pleinement dans l'actualisation de la vie et de la culture françaises au Québec $^{13}$.» Le sentiment d'appartenance était également complet et était favorisé par les luttes scolaires. Par contre, après 1930, l'espace social et linguistique des francophones commence à se modifier sous l'effet d'une accélération de l'industrialisation et de l'urbanisation. Le cultivateur se fait ouvrier en déménageant à la ville. D'une vie rurale française, il passe à une vie urbaine où la langue et la culture anglaises dominent. «Nous perdons notre appartenance sociétale traditionnelle et notre assise culturelle [. .] le groupe majoritaire définit de plus en plus les modèles d'attitudes et de comportements qui assurent un meilleur statut socio-économique ${ }^{14}$.» Ces phénomènes sont plus importants à partir de la décennie de 1960. Les francophones deviennent alors davantage minoritaires, puisqu'ils ne vivent plus dans des milieux homogènes de langue française.

Dans le chapitre suivant, Bernard se penche sur la situation économique des Franco-Ontariens. Il analyse leur profil professionnel, leur sous-scolarisation et leur revenu. Il conclut que «les inégalités sociales à l'intérieur de la communauté franco-ontarienne sont aussi flagrantes que les inégalités ethniques ${ }^{15}$ ", ce qui empêche une prise de conscience de classe susceptible de se transformer en prise de conscience ethnique.

\footnotetext{
${ }^{13}$ Ibid., p. 54.

${ }^{14}$ Ibid., p. 61.

${ }^{15}$ Ibid., p. 69.
} 
Le chapitre cinquième, qui porte sur l'identité francoontarienne, constitue le cœur de l'essai. «Nous ne sommes pas programmés pour être Franco-Ontariens», affirme d'emblée Bernard ${ }^{16}$. L'identité franco-ontarienne est un construit, à la base duquel se trouve la langue française, qui s'insère dans un ensemble d'éléments économiques, sociaux, culturels, et est affecté par des pratiques comme l'exogamie. Dans ce contexte, le groupe franco-ontarien devient davantage minoritaire et la langue française ne semble plus représenter un élément culturel central pour l'ensemble de la collectivité:

Si des Franco-Ontariens ont lutté pour définir et actualiser des idéologies de survivance et d'épanouissement de la langue et de la culture françaises, d'autres vont aujourd'hui se donner des instruments et des institutions, ou vont simplement vivre des situations d'interactions sociales et d'intégration structurelle, qui favoriseront l'actualisation des idéologies culturelles et linguistiques axées sur le bilinguisme. Cette situation conduira éventuellement à une assimilation culturelle plus poussée et plus rapide, qui accentuera la minorisation des francophones et la perte des éléments de différenciation ethnique traditionnelle ${ }^{17}$.

Les luttes pour obtenir des institutions et des services de langue française se butent ainsi à des transformations sociétales et à une mutation des représentations symboliques qui rendent presque impossible le maintien de la langue française en Ontario.

En épilogue, Bernard prédit que l'établissement des écoles secondaires de langue française et l'obtention de services gouvernementaux en français n'empêcheront pas les transferts linguistiques vers l'anglais, la complétude institutionnelle n'assurant plus la survie linguistique et culturelle de la communauté franco-ontarienne.

Les perspectives d'avenir ne sont pas reluisantes. Les individus sont de plus en plus marqués par les nouvelles valeurs d'actualisation de soi, de matérialisme, d'individualisme, de dégagement

\footnotetext{
${ }^{16}$ Ibid., p. 72.

${ }^{17}$ Ibid., pp. 98-99.
} 
politique et ethnique, c'est-à-dire des valeurs, centrées sur le rapport à l'environnement et au corps, qui entrent en contradiction avec certains aspects du développement d'une communauté franco-ontarienne. Les responsables des institutions ethniques de socialisation devront trouver les dispositions pour concilier les intérêts individuels et collectifs différents ${ }^{18}$.

En 1996, Bernard publie la seconde édition de De Québécois à Ontarois. Pour l'essentiel, il ne modifie pas son essai, se contentant de mettre à jour les tableaux et de donner de nouveaux titres à ses chapitres. Il scinde aussi en trois le dernier chapitre pour faire ressortir davantage les phénomènes linguistiques. "La langue française en Ontario» devient ainsi «Les Franco-Ontariens: un groupe linguistique», "Minorisation et bilinguisation", "La secondarisation du français». Le chercheur est encore plus pessimiste que huit ans plus tôt:

L'Ontario français est à bout de souffle, malade de son assimilation galopante, de sa fécondité fléchissante et de son vieillissement inquiétant. Les misères françaises ne s'arrêtent pas là. La francophonie ontarienne est aussi malade de sa francité qui s'étiole, de sa langue qui s'abâtardit et de son bilinguisme culturel qui prend les allures d'un cheval de Troie ${ }^{19}$.

De Québécois à Ontarois est l'œuvre d'un sociologue qui a mis tout son savoir au service des études francoontariennes. La force de l'essai est en même temps sa faiblesse, Bernard utilisant plusieurs théories qui ne se fondent pas en un tout harmonieux. Comme l'ont écrit Linda Cardinal, Jean Lapointe et Joseph-Yvon Thériault au sujet de la première édition, « $R$. Bernard fait appel à un certain nombre d'apports théoriques qui ne sont pas toujours convaincants, car le genre utilisé ne lui permet pas de les développer suffisamment ${ }^{20} . "$ Toutefois, l'ouvrage constitue une pierre blanche et, en 1991, l'Institut franco-ontarien

${ }^{18}$ Ibid., p. 123.

${ }^{19}$ Roger Bernard, De Québécois à Ontarois, Ottawa, Le Nordir, 1996, p. 9.

${ }^{20}$ Linda Cardinal et al., État de la recherche sur les communautés francophones hors Québec 1980-1990, Ottawa, Centre de recherche en civilisation canadienne-française de l'Université d'Ottawa, 1994, p. 87. 
décerne à Roger Bernard le prix Omer-Legault pour le livre le plus méritoire sur l'Ontario français.

\section{III - L'identité BiLINGUE}

La question de la langue occupe une position primordiale dans l'essai de Roger Bernard. C'est là qu'il développe pour la première fois la thèse de l'identité bilingue des Franco-Ontariens qui s'énonce comme suit: pendant les décennies de 1970 et de 1980 , le bilinguisme est devenu l'élément central de la culture franco-ontarienne. Ce bilinguisme accorde une place de plus en plus importante à l'anglais, le français se trouvant relégué au second plan. Dans une série de publications subséquentes à la première édition de De Québécois à Ontarois, le sociologue affine sa thèse. À cette époque, plus précisément en 1990, il devient professeur à la faculté des sciences de l'éducation de l'Université d'Ottawa; il dirige également la recherche de "Vision d'avenir», un grand projet national de cueillette et d'analyse de données sur les francophones du Canada, ce qui lui fournit l'occasion de tester ses hypothèses à l'extérieur de l'Ontario.

«Vision d'avenir» est une initiative de la Fédération des jeunes Canadiens français, dont les dirigeants veulent comprendre la situation dans laquelle évoluent les francophones canadiens et «identifier les éléments essentiels pour assurer le plein épanouissement des communautés acadiennes et francophones et celui des jeunes en particulier ${ }^{21}{ }$. Le livre 1, intitulé Le Déclin d'une culture, contient une présentation des grands courants de la recherche dans les années 1980, une analyse des sociétés francophones minoritaires et une bibliographie. Bernard y propose une série de principes pour un projet de communauté canadiennefrançaise:

\footnotetext{
${ }^{21}$ Roger Bernard, dir., Le Déclin d'une culture. Recherche, analyse et bibliographie: francophonie hors Québec 1980-1989, Livre 1 du projet «Vision d'avenir», Ottawa, Fédération des jeunes Canadiens français, 1990, p. 8.
} 
- la dualité linguistique et culturelle du Canada exige une charte des droits collectifs

- la survie et l'épanouissement de la communauté canadiennefrançaise doivent constituer un objectif pour tous ses membres - la culture et la langue françaises doivent devenir une valeur fondamentale

- il doit y avoir distinction entre l'espace linguistique du français langue seconde et l'espace social du français langue maternelle

- la langue doit être centrée sur la culture; l'idée d'un bilinguisme culturel doit disparaître et être remplacée par l'idée d'un bilinguisme fonctionnel

- les éléments intellectuels de la culture doivent être stabilisés et matérialisés dans les bibliothèques, les monuments, l'architecture, les livres, la langue et l'histoire

- l'identité individuelle et la culture personnelle des membres d'une communauté minoritaire doivent être maintenues par un réseau d'institutions

- la survie et le développement du Canada français exigent la mise sur pied de structures et d'institutions plus restreintes, qui deviennent ainsi le cœur et l'âme des communautés

- l'école de langue et de culture françaises doit être le lieu d'interactions qui resserrent les liens des membres de la communauté

- le développement de la culture individuelle passe de plus en plus par les communications de masse

- les «agents de culturation» doivent garder vivante la culture canadienne-française et favoriser le développement culturel, ainsi que la participation des francophones à la création de leur culture et de leur communauté22.

Dans le livre 2, l'équipe de Bernard dresse, à partir des données quinquennales de Statistique Canada et d'autres sources, un portrait statistique de la francophonie canadienne en 1986, en remontant parfois jusqu'en 1951. Le constat qui s'en dégage confirme les recherches de Bernard sur l'Ontario: hors du Québec, l'assimilation linguistique des francophones se poursuit à un rythme accéléré, particulièrement dans le groupe d'âge des 15 à 24 ans. De plus, les effectifs chutent et les pyramides d'âge s'inversent. La ${ }^{22}$ Ibid., pp. 132-133. 
fécondité, elle, est en baisse, en nombres absolus et par rapport à la population anglophone. Les apports migratoires sont peu importants et l'exogamie a souvent pour conséquence l'anglicisation. Quant à la scolarisation des francophones, elle est considérablement plus basse que celle des autres groupes linguistiques et culturels. Enfin, l'écart des revenus persiste entre les francophones et les anglophones ${ }^{23}$.

Le livre 3 a pour sujet les comportements linguistiques et la conscience culturelle des jeunes Canadiens français. Bernard s'appuie sur une enquête réalisée auprès de quelque 4000 jeunes francophones hors Québec répartis dans plus de 75 établissements secondaires, collégiaux et universitaires. Les comportements des jeunes touchent la famille, l'école, les amis, le travail, les médias, les activités culturelles, ainsi que les attitudes et les perspectives d'avenir en regard de la langue et de la culture françaises. Il en ressort que le problème de la représentation symbolique est profond, les jeunes ne se reconnaissant pas dans les médias, où dominent la langue et la culture de la majorité. Toutefois, les jeunes Canadiens français ont les mêmes attitudes que les jeunes Québécois en ce qui a trait à la valorisation du français ${ }^{24}$. La conclusion n'est pas des plus encourageantes: «Si le passé est garant de l'avenir, les présages sont tristes et lourds de conséquences. Au Canada, en situation minoritaire, ce n'est pas l'assimilation des francophones qui surprend, mais la survivance ${ }^{25} . "$

${ }^{23}$ Roger Bernard, dir., Le Choc des nombres. Dossier statistique sur la francophonie canadienne 1951-1986, Livre 2 du projet «Vision d'avenir», Ottawa, Fédération des jeunes Canadiens français, $1990,310 \mathrm{p}$.

${ }^{24}$ Une équipe de chercheurs québécois avait déjà réalisé une enquête semblable auprès de 5000 jeunes. Voir Uli Locher, Conscience linguistique des jeunes Québécois IV. Étude comparative du vécu et de la perception du fait français dans les écoles françaises et anglaises, Québec, Éditeur officiel, 1983, 158 p.; Les Jeunes et la langue (tome 1). Usages et attitudes linguistiques des jeunes faisant leurs études en français, Québec, Conseil de la langue française, 1993, 215 p.

${ }^{25}$ Roger Bernard, dir., Un avenir incertain. Comportements linguistiques et conscience culturelle des jeunes Canadiens français, Livre 3 du projet «Vision d'avenir", Ottawa, Fédération des jeunes Canadiens français, 1991, p. 235. 
Parallèlement à cette recherche commanditée, le sociologue continue à préciser sa pensée dans des articles. Dans son optique, l'idéal de beaucoup de Franco-Ontariens est de comprendre l'anglais, de le parler et de l'écrire comme les anglophones. Le français est de plus en plus restreint à la vie privée, à la famille et à l'école, pendant que l'anglais devient la langue publique. La francité est alors légitimée par le bilinguisme et n'est pas en elle-même une valeur fondamentale. Le Franco-Ontarien est un francophone bilingue; il fréquente une école de langue française, mais celle-ci est, dans les faits, bilingue et ne joue plus un rôle de reproduction culturelle. Dans plusieurs milieux minoritaires, elle devient même une école d'immersion au service de la majorité de langue anglaise. Il y a, par conséquent, une diminution de l'emploi du français, souvent restreint à la relation maître-élève, une simplification de la langue d'enseignement et une modification de la pédagogie pour tenir compte du niveau de compétence linguistique des élèves. Les effets sont contraires aux buts de l'école francoontarienne. L'élève ne peut améliorer sa langue maternelle, puisqu'il ne l'utilise que sporadiquement et il éprouve des difficultés à bien maîtriser sa langue seconde parce qu'il y a immixtion d'une langue à l'autre ${ }^{26}$.

En milieu minoritaire, le français est souvent une langue de traduction qui a comme point de départ l'anglais. Inconsciemment, cette langue «se transforme subrepticement en langue première, celle qui exprime les réalités fondamentales de la vie, celle dont les mots portent une charge émotive, celle qui baigne dans une culture et une histoire [. .] Molière est rentré à Versailles ${ }^{27}$." Apparaît alors un «je» biculturel qui fait partie de la nature intérieure de la personne, qui touche les fondements mêmes

${ }^{26}$ Id., «Molière est rentré à Versailles", dans Gérard Bouchard, dir., La Construction d'une culture. Le Québec et l'Amérique française, Sainte-Foy, Les Presses de l'Université Laval, 1993, p. 330.

${ }^{27}$ Id., "Réflexions critiques d'un chercheur», p. 338. 
de la personnalité et qui établit les assises de l'identité individuelle ${ }^{28}$.

\section{IV - EXogamiE ET TRANSFERTS LINGUISTIQUES}

En 1993, Roger Bernard devient membre de la Société Charlevoix. Dans le cadre de cette amicale vouée à l'étude de l'Ontario français, il rédigera trois articles sur les thèmes de l'exogamie et des transferts linguistiques, puisant sa matière dans les données de l'enquête "Vision d'avenir" et dans le recensement fédéral de 1991. Sa méthodologie est rigoureuse: il travaille à partir d'échantillons qu'il teste par une série d'hypothèses. Dans le premier Cahier Charlevoix, il prend beaucoup de soin à replacer l'exogamie dans l'ensemble des facteurs démographiques et sociaux qui en favorisent la montée. D'une société traditionnelle, rurale et catholique, les francophones canadiens sont passés à une société moderne, urbaine et industrielle, où leur condition de minoritaires devient plus aiguë. L'exogamie cesse d'être un tabou, ce qui se traduit par une hausse importante du phénomène: en 1991, plus du tiers des époux et des épouses de langue maternelle française vivant à l'extérieur du Québec choisissent un partenaire anglophone. Les taux d'exogamie varient considérablement d'une province à l'autre, selon le poids démographique qu'y occupent les francophones, mais partout l'exogamie est une cause de "bilinguisation» et d'anglicisation ${ }^{29}$.

Bernard poursuit et nuance cette analyse dans le deuxième Cahier Charlevoix en se concentrant cette fois sur les transferts linguistiques dans les foyers mixtes. Il

\footnotetext{
${ }^{28}$ Id., "Du social à l'individuel. Naissance d'une identité bilingue», dans Jocelyn Létourneau, dir., La Question identitaire au Canada francophone. Récits, parcours, enjeux, hors-lieux, Sainte-Foy, Les Presses de l'Université Laval, 1994, pp. 156-163.

${ }^{29} \mathrm{Id}$., «Langue maternelle et langue d'usage dans les foyers mixtes. Les enjeux de l'exogamie", Cahiers Charlevoix, 1, Sudbury, Société Charlevoix et Prise de parole, 1995, pp. 241-289.
} 
montre hors de tout doute que l'exogamie est une cause majeure d'anglicisation. Ainsi, à l'extérieur du Québec, dans les foyers mixtes, neuf époux francophones sur dix et huit épouses francophones sur dix utilisent l'anglais dans leur ménage. Les mères réussissent mieux que les pères à transmettre le français comme langue maternelle unique à leurs enfants, mais, en général, l'anglais devient la langue d'usage. Cela constitue un échec, puisque les parents "croient [. .] que le maintien du français dans des conditions adverses est une question de volonté du couple et de convictions personnelles, qu'ils réussiront où les autres ont échoué ${ }^{30}$ \%. C'est ainsi qu'une décision individuelle, celle de former un couple avec une personne d'un autre groupe linguistique, influence le devenir collectif d'une société.

La Société Charlevoix publie de façon posthume le dernier article de Roger Bernard. Il s'agit d'une étude sur la langue de communication que les enfants utilisent avec leurs parents dans les foyers mixtes. Sans surprise, le sociologue constate que l'anglicisation des échanges est à l'œuvre: «Si le bilinguisme à géométrie variable caractérise les foyers exogames francophones, c'est l'unilinguisme qui caractérise les foyers exogames anglophones ${ }^{31}$." Dans le premier cas, les comportements linguistiques sont complexes, le choix de la langue d'usage dépendant essentiellement du niveau de bilinguisme des interlocuteurs et de l'interprétation du contexte de l'échange. Les enfants utilisent un peu plus souvent le français avec la mère francophone, surtout lorsqu'ils sont très jeunes. "Il est fort probable, écrit Bernard, que dans la petite enfance, la mère francophone réussisse à maintenir un statut prioritaire au

${ }^{30} \mathrm{Id}$., «Transferts linguistiques et anglicisation des francophones. Les enjeux de l'exogamie au Canada", Cahiers Charlevoix, 2, Sudbury, Société Charlevoix et Prise de parole, 1997, p. 245.

${ }^{31}$ Id., "Langue d'usage avec les parents à l'intérieur des foyers mixtes francophones. Les enjeux de l'exogamie», Cahiers Charlevoix, 4, Sudbury, Société Charlevoix et Prise de parole, 2000, p. 22. 
français, alors que la famille occupe une place prioritaire dans la vie des jeunes enfants ${ }^{32}$.» La situation se modifie à l'adolescence, lorsque l'influence des parents diminue, surtout dans les milieux où la vitalité ethnolinguistique est faible. En général, c'est à la fin de l'adolescence que se fait le passage d'un bilinguisme franco-dominant à un bilinguisme intégral ou anglo-dominant. La tendance vers l'anglicisation est encore plus forte avec le père anglophone. «En milieu minoritaire, conclut Bernard, il est très difficile de maintenir l'usage habituel du français dans les échanges entre les parents et les enfants, en dépit du fait que les foyers mixtes avaient un préjugé très favorable envers la langue et la culture françaises et que les parents avaient choisi de s'intégrer à la communauté canadiennefrançaise ${ }^{33}$."

\section{V - LE SPÉCIALISTE DES MIGRATIONS}

Les recherches de Roger Bernard sur l'exogamie, les transferts linguistiques et la naissance d'une identité bilingue lui confèrent un rôle de personnage public, ce qui a rejeté dans l'ombre son œuvre de pionnier dans le domaine des études migratoires, un sujet qui le fascinait et auquel il avait consacré sa thèse de doctorat à l'université McGill en 1987. Dans ce travail, publié quatre ans plus tard, il demeure fidèle à l'approche weberienne, en se demandant comment se prirent les décisions individuelles d'émigrer du Québec vers le nordest de l'Ontario: "Comment se fait-il que des colons aient préféré défricher des terres au Lac Sainte-Thérèse plutôt qu'au Lac Saint-Jean? Par quelles circonstances ou quels choix idéologiques ces migrants québécois arrivent-ils à prendre la décision de s'enraciner dans une région considérée comme inhospitalière et périphérique ${ }^{34}$ ?»

${ }^{32}$ Ibid., p. 28.

${ }^{33}$ Ibid., p. 43.

${ }^{34}$ Roger Bernard, Le Travail et l'espoir. Migrations, développement économique et mobilité sociale Québec/Ontario 1900-1985, Hearst, Le Nordir, 1991, p. 15. 
Le sujet est doublement original: il constitue une première en études franco-ontariennes et, du point de vue méthodologique, il n'a son pareil nulle part ailleurs, Bernard s'étant servi de questionnaires. En accordant ainsi toute la place qu'il mérite à l'acteur social, le sociologue poursuit, sans le savoir, la même démarche que les historiens des migrations nord-américaines ${ }^{35}$. En outre, comme eux, il s'attache à situer les mouvements de population dans les contraintes structurelles de la socio-économie et à expliquer la complexité de l'interaction entre les acteurs migrants et les dites contraintes.

L'ouvrage comprend trois parties: «Le sentier migratoire Québec / Hearst", «Le contexte économique, social et historique des migrations entre l'Ontario et le Québec", "Mobilité et intégration des migrants". La première partie s'ouvre sur des considérations théoriques. Encore une fois, Bernard fait appel à Durkheim et à Weber, mais il s'appuie aussi sur les écrits de Raymond Boudon pour orienter son questionnement. En ce qui a trait aux migrations proprement dites, il est influencé par deux approches qu'il veut complémentaires: l'approche fonctionnaliste et l'approche globalisante du système mondial. Il fait ensuite appel aux deux hypothèses principales avancées pour expliquer les migrations entre le Québec et l'Ontario: la motivation économique, les facteurs socioculturels de la prise de décision, ce qui l'amène à présenter une douzaine de propositions pour échafauder sa problématique. S'étant ainsi muni d'outils conceptuels et méthodologiques, il peut alors faire œuvre d'historien et décrire le sentier migratoire Québec/Hearst, en faisant ressortir le rôle du gouvernement de l'Ontario ainsi que de l'ouverture du chemin de fer, des chantiers forestiers et des exploitations minières. Il montre

${ }^{35}$ John Bodnar, The Transplanted. A History of Immigrants in Urban America, Bloomington, Indiana University Press, 1985, en particulier pp. 1-56; Andrée Courtemanche et Martin Pâquet, dir., Prendre la route. L'expérience migratoire en Europe et en Amérique du Nord du XIV $V^{e}$ au $X X^{e}$ siècle, Hull, Vents d'Ouest, 2001, pp. 13-21. 
comment, à leur arrivée en Ontario, les migrants pratiquent une économie agroforestière qui n'est pas fondamentalement différente de celle du Québec. Il trace ensuite le profil du mouvement migratoire: un Québécois sur cinq est venu avant 1940, alors que les quatre autres sont arrivés à chacune des décennies entre 1940 et 1980 . Les lieux d'origine varient selon la période de migration. Avant 1930, deux migrants sur trois proviennent de la Beauce, principalement des paroisses de l'arrière-pays près de la frontière avec les États-Unis. Cette proportion baisse à 50\% pendant la crise économique; des Montréalais tentent alors l'aventure vers Hearst et ses environs. De 1940 à 1970, l'Abitibi-Témiscamingue et le Bas-Saint-Laurent/Gaspésie envoient aussi un nombre important de migrants vers la région de Hearst. Finalement, dans les années 1970, près du tiers des migrants provient d'autres régions que celles précitées.

L'attrait d'un emploi est la cause la plus souvent évoquée par les migrants pour expliquer leur décision de partir. Quant au choix de Hearst, il s'explique surtout par la présence d'une connaissance ou d'un membre de la famille déjà installé dans la région. La décision d'émigrer se prend rapidement, en général lors de la visite d'un individu déjà rendu en Ontario. Bernard commente: «Nous sommes loin des analyses complexes des coûts et bénéfices que les modèles explicatifs imputent aux migrants et plus particulièrement aux migrants interprovinciaux. Selon notre enquête, l'analyse n'est pas complexe parce qu'ils n'ont pas grand-chose à perdre et beaucoup à gagner ${ }^{36}$.» Et, contrairement à ce qu'en dit l'historiographie traditionnelle, les prêtres colonisateurs n'ont pas joué un rôle très important dans la prise de décision.

Selon Bernard, la continuité socioculturelle est l'élément le plus frappant du sentier migratoire Québec/Hearst,

\footnotetext{
${ }^{36}$ Bernard, Le Travail et l'espoir, p. 61.
} 
les migrants s'installant dans des lieux de destination qui ressemblent aux lieux d'origine. La région de Hearst constitue donc «un prolongement naturel du milieu québécois, prolongement qui à son tour facilite le passage d'une province française à une province anglaise ${ }^{37}$ ", la frontière interprovinciale s'estompant dans l'esprit des migrants.

Dans la deuxième partie du livre, Bernard utilise une approche macroscopique en s'appuyant sur des statistiques et des travaux publiés. Tour à tour, il se penche sur l'émigration des Québécois, sur les migrations interprovinciales et sur le peuplement du nord de l'Ontario. Ici, il est clair qu'il adopte la thèse fonctionnaliste, la migration des travailleurs s'expliquant, selon lui, par un «ensemble de situations provoquées par des actions dans les sociétés situées à chaque extrémité du sentier migratoire ${ }^{38}$ ).

La troisième partie du Travail et l'espoir porte dans un premier temps sur la migration en tant que tremplin de mobilité sociale et professionnelle. Les migrants, qui proviennent de milieux sociaux humbles et qui sont sousscolarisés, connaissent des débuts modestes dans la région de Hearst, quand ils ne sont pas carrément pauvres. «Il n'y a rien d'étonnant à la faible scolarisation et à la reproduction de cette situation d'une génération à l'autre, écrit Bernard. Avec la migration, le problème se déplace, et les conditions du monde du travail de la région de destination, centré sur l'exploitation des ressources forestières et d'une agriculture vivrière, ne provoqueront pas de changements majeurs en regard des besoins de scolarisation et de spécialisation des travailleurs ${ }^{39}$ ». Les migrants proviennent de milieux ruraux et ils s'installent dans un milieu rural. Cependant, en déménageant du Québec vers l'Ontario, ils travaillent davantage comme bûcherons, tout en continuant à exploiter une petite ferme. Ce mode de vie

${ }^{37}$ Ibid., p. 66.

${ }^{38}$ Ibid., p. 148.

${ }^{39}$ Ibid., p. 159. 
agroforestier représente une transition vers des emplois à temps plein dans les scieries et dans l'industrie du bois d'œuvre. Les migrants continuent toutefois d'appartenir aux mêmes strates socio-économiques qu'auparavant. «La mobilité sociale échappe donc aux migrants. Tout se passe comme si la migration ne brisait pas certaines régularités sociales établies par le milieu social et familial et transmises de génération en génération ${ }^{40}$." Pourtant, Bernard ne peut s'empêcher de noter que les migrants ne visaient pas la mobilité ascendante: ils cherchaient avant tout à se procurer un emploi régulier, bien rémunéré, et des possibilités d'avancement pour leurs enfants. De plus, si la migration n'a pas amené de mobilité sociale, elle a néanmoins empêché la régression et rétabli la répartition des avantages économiques dans les deux sociétés.

L'intégration des migrants dans la région de Hearst est favorisée par les caractéristiques socio-économiques et socioculturelles du milieu d'accueil, qui ressemble au milieu de départ. Au fil du peuplement par des francophones, la région devient un "petit Québec" et le sentier migratoire continu raffermit les liens des communautés à l'origine et à la destination. Cette intégration se fait d'autant plus facilement que plusieurs migrants ont l'impression de ne pas avoir eu le choix de partir ou non; il leur faut donc s'adapter coûte que coûte. Les migrants bénéficient en outre d'un réseau familial et institutionnel en grande partie de langue française. En plus, ils arrivent jeunes en Ontario. Et, lorsqu'ils vieillissent, leurs enfants demeurent en général dans la région de Hearst ou du moins dans le nord de l'Ontario. Ces facteurs expliquent pourquoi les migrants sont contents, a posteriori, d'avoir pris la décision de quitter les campagnes québécoises pour la région de Hearst.

Comme pour De Québécois à Ontarois, on peut reprocher à Bernard d'avoir voulu prendre la bouchée trop grosse dans Le Travail et l'espoir. Il fait ainsi appel à deux to Ibid., p. 192. 
ROGer BERnARD, INTELLECTUEL DE L'ONTARIO FRANÇAIS

théories des migrations qui ne s'accordent pas et qui sont même contradictoires sous certains aspects. De plus, l'ouvrage n'est pas sans problème méthodologique: l'échantillon est trop petit par endroits et, plus fondamentalement, Bernard n'a interrogé que les individus qui sont restés dans la région de Hearst. S'il avait tenu compte de ceux qui sont repartis, il serait sans doute parvenu à des conclusions différentes sur la facilité de l'intégration. Toutefois, au risque de nous répéter, c'est un travail de pionnier sur un sujet trop peu étudié.

\section{VI - Le Savant ET LE Militant}

Nous avons vu en début d'article que les travaux de Roger Bernard suscitent des discussions et que ses relations avec les militants franco-ontariens sont parfois difficiles. Pourtant, lui-même est engagé dans la survie et le développement de l'Ontario français. Il y a certes des tensions entre Roger Bernard le savant et Roger Bernard le militant, mais il n'y a pas de contradiction. Le premier est amoureux de la vérité et réticent face au second, de qui il exige la même probité intellectuelle. Bernard croit que la "bonne» recherche peut avoir une fonction sociale. Ainsi souhaite-t-il la création d'un institut de recherche et d'analyse sociales qui mènerait des recherches fondamentales pour les communautés francophones, notamment sur l'éducation en milieu minoritaire, qui l'inquiète beaucoup: «Nous ne connaissons plus l'étudiant [sic] à qui nous nous adressons $s^{41}$." Selon lui, l'école devrait, dans un premier temps, provoquer un ressourcement aux valeurs fondamentales de la culture canadienne-française, la culture des "vieux», et, dans un deuxième temps, l'élaboration de modèles culturels franco-ontariens modernes qui s'insèrent dans l'univers culturel des jeunes. Il est aussi très critique

${ }^{41}$ Roger Bernard, «Proposition pour la création d'un institut de recherche et d'analyse sociales», dans Yolande Grisé, dir., États généraux de la recherche sur la francophonie à l'extérieur du Québec, Ottawa, Actexpress, I995, p. 222. 
envers les travaux de la Commission royale sur l'éducation en Ontario, qui confond l'éducation et l'apprentissage en accordant une trop grande importance à la technologie. Défendant une conception humaniste de l'éducation, il privilégie la relation maître-élève et la relation entre élèves.

Mais c'est dans l'affaire Montfort que l'engagement de Roger Bernard se manifeste avec le plus d'éclat. En effet, en juillet 1998, il présente un bref devant la Cour divisionnaire de l'Ontario pour la convaincre de renverser la décision de la Commission de restructuration des services de santé, qui préconise des fusions d'établissements préjudiciables au caractère francophone de l'hôpital Montfort. Il considère ce manifeste comme "la quintessence de son cheminement intellectuel ${ }^{42}$ ».

Avec éloquence, Bernard explique aux juges le fonctionnement du mécanisme qui permet à la société francoontarienne de survivre dans un milieu anglophone majoritaire et il montre comment les modifications du statut de l'hôpital Montfort menacent la vitalité linguistique et culturelle de l'Ontario français: le francophone qui fréquentera l'hôpital bilingue, utilisera l'anglais pour obtenir promptement des soins de santé. «Il reconnaîtra rapidement qu'il se trouve dans une institution à caractère anglo-dominant [. . ] et il sentira qu'il est normal et naturel d'utiliser l'anglais si les circonstances de l'échange l'exigent [. . ]. La pratique et l'habitude lui enseigneront qu'en utilisant l'anglais il ne se trompe pas ${ }^{43}$." La disparition de Montfort comme hôpital général représente beaucoup plus que la perte d'un service, les Franco-Ontariens perdant une institution qui incarne et évoque la présence française en Ontario. «Après la restructuration, il (l'hôpital Montfort) deviendra le symbole de la défaite d'une communauté qui n'a pas été capable de garder intact son réseau institutionnel, le symbole d'une commu-

\footnotetext{
${ }^{42}$ Robert Yergeau, "Préface», Bernard, À la défense de Montfort, p. 23.

${ }^{43}$ Bernard, À la défense de Montfort, p. 43.
} 
nauté qui se fragilise. ${ }^{44}{ }^{»}$ Car la complétude institutionnelle se reconnaît davantage à la variété des institutions qu'à leur nombre. «Une communauté dynamique dispose d'une bonne vitalité si c'est une communauté où "on peut naître, vivre et mourir" [ . .]. Sans contredit, la transformation de Montfort risque de rétrécir sérieusement le réseau institutionnel franco-ontarien et, conséquemment, d'affaiblir l'ensemble de la communautét ${ }^{45}$.» Dans la lutte pour Montfort, conclut Bernard, les Franco-Ontariens doivent résister jusqu'à leur dernier souffle, puisque la disparition de cette institution entraînerait leur déclin.

À sa grande surprise, les juges se sont inspirés de son intervention dans leur décision historique: «Le témoignage du Dr. [sic] Bernard établit que, dans un milieu bilingue, le français est condamné à devenir la deuxième langue des Franco-Ontariens [. .]. Dans un contexte minoritaire, le bilinguisme risque de devenir la première étape de l'assimilation d'un groupe minoritaire bilingue ${ }^{46}$.» Roger Bernard le savant et Roger Bernard le militant s'étaient rejoints.

\section{Conclusion}

De Departure-Lake, où habite sa famille lorsqu'il naît, à Alexandria, où il décède, en passant par Toronto, Hallébourg, Hearst et Ottawa, où il vit et travaille, la trajectoire de Roger Bernard suit les contours de l'Ontario français. Dans ses écrits, il est avare de détails sur sa vie personnelle, mais ses idées sur la société à laquelle il appartient sont autant ancrées dans sa condition de minoritaire que dans sa pratique sociologique. Cet ancrage est à la fois producteur de richesse et de misère intellectuelles, mais c'est justement ce double enracinement qui lui permet de critiquer la société franco-ontarienne.

\footnotetext{
${ }^{44}$ Ibid., pp. $48-49$.

${ }^{45}$ Ibid., pp. 52-53.

${ }^{46} \mathrm{http}: / /$ radio-Canada.ca/regions/est-ontarien-outaouais/dossiers/montfort/ montfort_fre 1.htm
} 


\section{YvES FRENETTE}

Ses idées sont controversées, mais de la controverse jaillissent les progrès de la recherche. Ainsi, il n'est pas possible d'étudier la société franco-ontarienne contemporaine sans tenir compte de la thèse de l'avènement d'une identité bilingue. Bernard avance d'abord cette thèse dans le cadre de son mémoire de maîtrise portant sur trois communautés de l'Ontario. Il la développe ensuite dans son essai De Québécois à Ontarois pour expliquer la mutation identitaire des Franco-Ontariens, lui ajoutant le corollaire de la "secondarisation" de la langue française. Ce faisant, le sociologue contribue à la création d'outils conceptuels et théoriques d'analyse des groupes minoritaires. Jamais, cependant, il ne perd de vue les êtres de chair et d'os qui évoluent au sein de ces groupes et les problèmes concrets qui se posent à eux dans leur vie quotidienne. S'il fustige à l'occasion les militants de la survivance, c'est pour que ceux-ci soient lucides dans leur action. C'est là le signe d'un engagement profond envers l'Ontario français. 


\section{L'CEuvre de Roger Bernard}

\section{LIVRES}

1988 De Québécois à Ontarois. La communauté francoontarienne. Hearst, Le Nordir, 1988, 189 p. (Prix OmerLegault). Deuxième édition revue, corrigée, mise à jour et augmentée, Ottawa, Le Nordir, 1996, 187 p.

1990 Le Déclin d'une culture. Recherche, analyse et bibliographie. Francophonie hors Québec 1980-1989. Livre I. Ottawa, Fédération des jeunes Canadiens français, 1990, $198 \mathrm{p}$.

1990 Le Choc des nombres. Dossier statistique sur la francophonie canadienne, 1951-1986. Livre II. Ottawa, Fédération des jeunes Canadiens français, 1990, $311 \mathrm{p}$.

1991 Un avenir incertain. Comportements linguistiques et conscience culturelle des jeunes Canadiens français. Livre III. Ottawa, Fédération des jeunes Canadiens français, $1991,280 \mathrm{p}$.

1991 Le Travail et l'espoir. Migrations, développement économique et mobilité sociale. Ontario / Québec 19001985. Ottawa, Le Nordir, 1991, 396 p. (Prix Le Droit).

1994 En collaboration avec Jocelyn Létourneau (dir.). La Question identitaire au Canada francophone: récits, parcours, enjeux, hors-lieux. Québec, Les Presses de l'Université Laval, 1994, 292 p.

1998 Le Canada français: entre mythe et utopie. Ottawa, Le Nordir, 1998, 238 p.

2000 À la défense de Montfort. Ottawa, Le Nordir, 2000, 57-2 p.

\section{Chapitres de livre}

1978 «Comportement linguistique des Canadiens français dans trois villes du Nord-Est ontarien", dans Actes de colloque I/Langue maternelle, langue première de communication, Sudbury, Institut franco-ontarien, 1978, pp. 109-133. 
1989 "La Bilinguisation de la culture franco-ontarienne", dans Actes du colloque Proaction: réseau de vie en français, Cornwall, 1989, pp. 2-7.

1989 «Les Franco-Ontariens: une communauté ethnoculturelle», dans les Actes du colloque: les voies de l'avenir franco-ontarien, Ottawa, Association canadiennefrançaise de l'Ontario, 1989, pp. 9-14.

1993 «Molière est rentré à Versailles», dans Gérard Bouchard et Serge Courville (dir.), La Construction d'une culture. Le Québec et l'Amérique française, Québec, Les Presses de l'Université Laval, 1993, pp. 329-342.

1993 «Culture et identité franco-ontariennes», dans JeanPierre Pichette (dir.), L'CEuvre du père Germain Lemieux, s.j. Bilan de l'ethnologie en Ontario français, Sudbury, Prise de parole et Centre franco-ontarien de folklore, 1993, pp. 449-462.

1994 «Comportements linguistiques et conscience culturelle des jeunes Canadiens français", dans Claude Poirier et al. (dir), Langue, espace, société. Les variétés du français en Amérique du Nord, Québec, Les Presses de l'Université Laval, 1994, pp. 319-334.

1994 «Du social à l'individuel: naissance d'une identité bilingue", dans Jocelyn Létourneau et Roger Bernard (dir.), La Question identitaire au Canada francophone: récits, parcours, enjeux et hors-lieux, Québec, Les Presses de l'Université Laval, 1994, pp. 155-163.

1994 «Les Enjeux de l'exogamie», dans Fédération des communautés francophones et acadienne / Commissariat aux langues officielles (dir.), Actes du mini-colloque national sur l'exogamie et les structures d'accueil des immigrants et immigrantes francophones, Ottawa, Commissariat aux langues officielles, 1994, pp. 3-12.

1995 «Proposition pour la création d'un institut de recherche et d'analyse sociales", dans Yolande Grisé (dir.), États généraux de la recherche sur la francophonie à l'extérieur du Québec, Ottawa, Les Presses de l'Université d'Ottawa, 1995, pp. 221-222. 
Roger Bernard, InTELlectuel de L'ONTARIo FRANÇAis

1995 «Langue maternelle et langue d'usage dans les foyers mixtes francophones: les enjeux de l'exogamie", dans Cahiers Charlevoix I. Etudes franco-ontariennes, Sudbury, Prise de parole et Société Charlevoix, 1995, pp. 241-289.

1995 «Réflexions critiques d'un chercheur», dans Jacques Cotnam, Yves Frenette et Agnès Whitfield (dir.), $L a$ Francophonie ontarienne: bilan et perspectives de recherche, Toronto, Le Nordir, 1995, pp. 327-340.

1997 «Transferts linguistiques et anglicisation des francophones: les enjeux de l'exogamie au Canada», dans Cahiers Charlevoix 2, Études franco-ontariennes, Sudbury, Prise de parole et Société Charlevoix, 1997, pp. 213-262.

1999 «Entrevue de Roger Bernard réalisée par François X. Chamberland", dans L'Ontario se racontre. De A à X. Entrevues radiophoniques réalisées et présentées par François-Xavier Chamberland, Toronto, Éditions du Gref, 1999, pp. 414-429.

1999 En collaboration avec Louis-Gabriel Bordeleau et Benoît Cazabon, «L'Éducation en Ontario français», dans Francophonies minoritaires au Canada: l'état des lieux, Moncton, Les Éditions de l'Acadie, 1999, pp. 435-473.

2000 «Langue d'usage avec les parents à l'intérieur des foyers mixtes francophones: les enjeux de l'exogamie», dans Cahiers Charlevoix 4, Études franco-ontariennes,

Sudbury, Prise de parole et Société Charlevoix, 2000, pp. 13-49.

2000 «Enjeux culturels du savoir: des idées sur le Canada français», dans Luc Rousseau et Yvon Gauthier (dir.), Actes de la $6^{e}$ Journée du savoir de l'ACFAS-Sudbury (19 mars 1999), Sudbury, Institut franco-ontarien, «Fleur-detrille», 2000, pp. 9-29.

200? En collaboration avec M. Saint-Germain, «Le Rôle des grandes théories éthiques dans la démarche de l'évaluation de projets éducatifs», Cédérom / Colloque international "Ethique et qualité dans l'évaluation", Université de Reims (1999). 


\section{ArTicles de PÉRIOdique}

1985 «L'Ontario français: pratiques ethniques et théories sociologiques", dans Revue de l'Université d'Ottawa, Ottawa, vol. 55, $\mathrm{n}^{\circ} 2,1985$, pp. 137-150.

1986 "Le Rôle social des institutions ethniques", dans Revue du Nouvel-Ontario, Sudbury, Institut franco-ontarien, $n^{0}$ 8, 1986, pp. 41-48.

1990 «Peuplement du Nord de l'Ontario", dans Revue du Nouvel-Ontario, Sudbury, Institut franco-ontarien, $\mathrm{n}^{\circ} 12$, 1990, pp. 15-40.

1996 «La Nouvelle Culture de l'évaluation: passage difficile de la modernité à la postmodernité», dans Mesure et évaluation en éducation, vol. 19, $\mathbf{n}^{\circ} 2,1996$, pp. 99-1 11.

1996 "Portrait démolinguistique de l'Ontario français", dans Revue du Nouvel-Ontario, Sudbury, Institut francoontarien, $\mathrm{n}^{\circ} 20,1996$, pp. 15-40.

1997 "Les Contradictions fondamentales de l'école minoritaire», dans Revue des sciences de l'éducation, vol. XXIII, no 3, 1997, pp. 509-526.

200? En collaboration avec D. Ducharme, "Communication Breakdowns: An Exploration of Contextualization in Native and Non-Native Speakers of French $»$, dans Journal of Pragmatics (An Interdisciplinary Monthly of Language Studies), Odense University (Danemark).

\section{Articles de magazine}

1975 "La Fin d'une langue», dans Boréal, Hearst, n², 1975, pp. 2-4.

1975 En collaboration avec René Guindon et Raymond Tremblay, "Le Professeur et la tâche de socialisation", dans Boréal, Hearst, $\mathrm{n}^{\circ}$ 2, 1975, pp. 74-78.

1976 "Entrevue avec René Brixhe», dans Boréal, Hearst, n 5 , 1976, pp. 72-84.

1985 "L'Autre Nord québécois", dans Liaison, Ottawa, n³7, 1985, pp. 26-27.

1988 "Hearst: Migrations et développement", dans Atmosphères, Hearst, $\mathrm{n}^{\circ}$ 1, 1988, pp. 7-17. 
1990 «La Situation linguistique des jeunes francophones dans les communautés minoritaires canadiennes", dans Éducation et francophonie, vol. XVIII, 1990, pp. 5-17.

1991 «Éduquer en français au Canada: conjoncture sociodémographique», dans Éducation et francophonie, Les Actes du $44^{e}$ congrès de l'ACELF, vol. XIV, numéro spécial, décembre 1991, pp. 20-25.

1992 «Le Bilinguisme et l'école minoritaire», dans Éducation et francophonie, actes du congrès, vol. XX, numéro spécial, novembre 1992, pp. 45-46.

1992 «L'École de langue française en milieu minoritaire: une analyse socio-démographique», dans Éducation et francophonie, vol. XX, nº 2, 1992, pp. 34-38.

1993 «La Culture éclatée», dans Liaison, Ottawa, $\mathbf{n}^{\circ} 73$, septembre 1993, pp. 12-14.

\section{COMPTES RENDUS}

1993 «Dean Louder (dir.), Le Québec et les francophones de la Nouvelle-Angleterre (Québec, PUL, 1991)", dans Revue d'histoire de l'Amérique française, Montréal, Institut d'histoire de l'Amérique française, vol. 47, n² 2, 1993, pp. 288-291.

1997 «Jacques Ardoino et René Lourau, Les Pédagogies institutionnelles (Paris, PUF, 1994)", dans Revue des sciences de l'éducation, vol. XXIII, nº 2, 1997, pp. 418-419.

\section{Articles et lettres dans des journaux}

1982 "L'Avenir de l'université dans le Nord-Est de l'Ontario", dans Le Nord, Hearst, 1982.

1982 «Le Contexte socio-économique de la nouvelle université du Nord-Est», dans Le Nord, Hearst, 1982.

1992 «Un processus croissant de minorisation", dans Le Devoir, cahier spécial "Francophonie canadienne", Montréal, 13 juin 1992, p. 5.

1995 «Les Enseignants dépossédés de l'enseignement», dans Le Droit, Ottawa, 24 mars 1995.

1995 «L'Université bilingue et la potion magique», dans Le Droit, Ottawa, 12 mai 1995. 